\title{
75. An Experimental Study of Instantaneous Electric Oscillations by Vacuum Tube.
}

By Yoshiro Ikeda, Etsuro Kato and Motokichi Mori.

Physical Institute, Hokkaido Imperial University, Sapporo.

(Rec. May 12, 1929. Comm. by T. TeradA, M.I.A., June 12, 1929.)

If we insert a horn gap in the secondary of a transformer or an induction coil, and adjust the distance of the gap, we can see a number of sparks or arcs grouped together in each cycle of the alternating electric current. By the spark and arc discharge there will be excited an instantaneous electric oscillation in the circuit, but when the frequency of the oscillation is very high, we cannot measure the electric oscillation by means of an ordinary oscillograph.") Therefore, if we want to investigate the instantaneous oscillation in such a circuit, we must use some apparatus which is sensitive enough to indicate the instantaneous state of the current. Moreover, it is evident that the electric conditions of the current must not be affected by inserting the apparatus. From this consideration, we have tried to insert a spectrum tube of helium of $14 \mathrm{~cm}$. length in series with the gap, and to take a photograph of the glow of the tube with a rotating film.

It may be supposed that the spectrum tube might cause some disturbances in the circuit, and diminish the potential difference at the gap. Fig. 1 shows the groups of the sparks in the case where the gap is connected directly to the secondary of a transformer (max. volts 70,000 , cycle 60 ) while Fig. 2 shows them, in the case where we have inserted the spectrum tube and kept the other conditions the same. The potential drop due to the existence of the tube was very small against the terminal voltage of the gap. The lower series of lines given in Fig. 2 shows the intermittent glows of the tube, which correspond to the sparks at the gap shown in the upper series. It is remarkable that when there appears an arc at the gap, the glow of the tube gives on the rotating film generally a continuous band and sometimes a band with lines, though the arc itself is not bright enough to be photographed by the rotating film, as shown in the Figs. 3 and 5 ; the latter shows the photograph taken in the case where we used,

1) The cathode ray oscillograph such as used by Rogowski, Arch.f. Elektrot., 14 (1925), 18 (1927), Naturwiss., 164 (1928), etc., enables us to measure an instantaneous electric state of $10^{-9}$ second, but it is not easy to be manipulated. 
as the high tension producer, an induction coil having a spark length of $30 \mathrm{~cm}$.

From the results of these preliminary experiment it may be stated that

(a) a convenient means of studying the instantaneous electric oscillation in a high tension circuit may be afforded by making use of the spectrum tube and rotating film ${ }^{1)}$; the resolving power of the oscillation being more than $20,0001 /$ sec. as shown in the Fig. 4.

(b) and that it would be instructive to study the sparking phenomena from the photographs taken by this method; at least, it would afford a useful method for studying the sparking phenomena of a horn gap used as an arrester.

The details and the results of further researches will be published later in an other place.

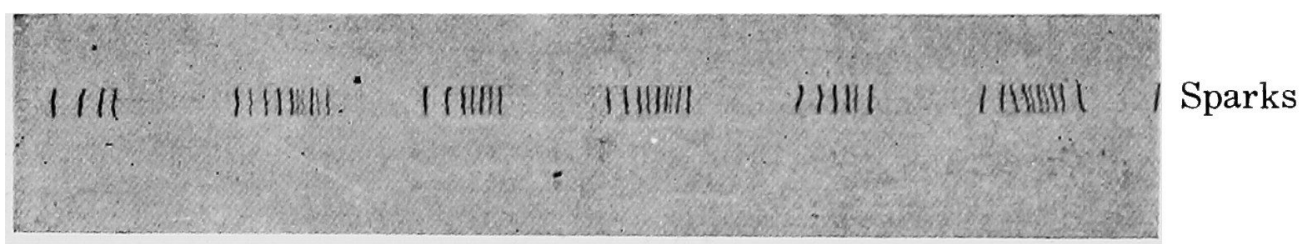

Fig. 1. Each two groups denote the sparks in one cycle. $\rightarrow t$ With a transformer, 60 cycles.

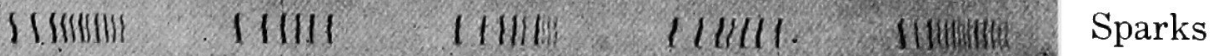

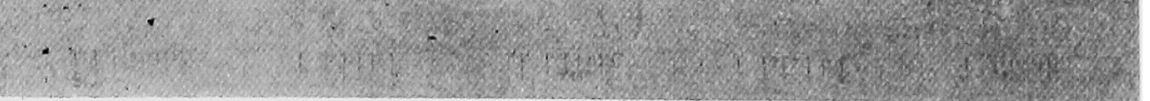

Glows of the tube

Fig. 2. With a transformer, 60 cycles.

$\rightarrow t$

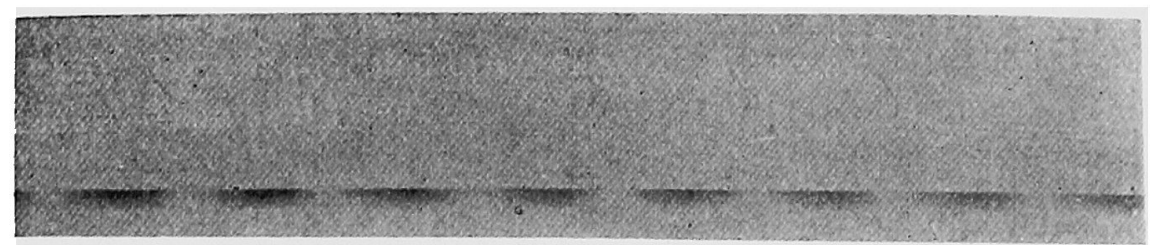

Sparks and arcs

Glows of the tube

Fig. 3. With a transformer, 60 cycles.

$\rightarrow t$

1) The so-called "Glimmlicht Oscillograph" can be used for the same purpose; the discussion about it is to be referred to the book, entitled "Empfindliche Galvanometer" by Werner, and also the original papers, Gehrcke, ZS. f. Instrkd. 25 (1905), Engelhardt und Gehrcke, Z. f. techn. Physik, 1925, F. Voltz Arch. f. Elektrotechnik, 9 (1920). 
No. 6.] An Experimental Study of Instantaneous Electric Oscillations.

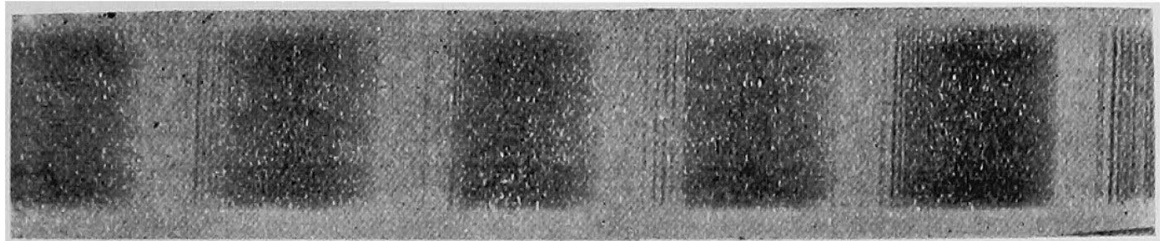

Glows of the tube

Fig. 4. With a transformer, 60 cycles.

$\rightarrow t$

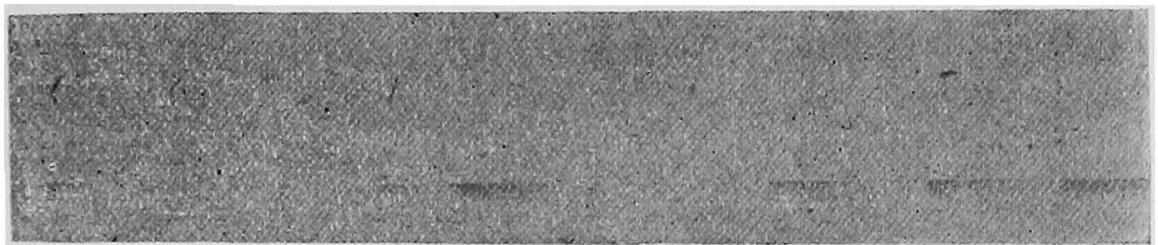

Sparks and arcs

Glows of the tube

Fig. 5. With an induction coil.

$\rightarrow t$ 